\title{
Physical basis of the 'magnification rule' for standardized Immunohistochemical scoring of HER2 in breast and gastric cancer
}

\author{
Andreas H. Scheel ${ }^{1 *}$, Frédérique Penault-Llorca², Wedad Hanna ${ }^{3}$, Gustavo Baretton ${ }^{4}$, Peter Midde ${ }^{5,6}$,
} Judith Burchhardt ${ }^{5}$, Manfred Hofmann ${ }^{5}$, Bharat Jasani ${ }^{7}$ and Josef Rüschoff ${ }^{5,7}$

\begin{abstract}
Background: Detection of HER2/neu receptor overexpression and/or amplification is a prerequisite for efficient anti-HER2 treatment of breast and gastric carcinomas. Immunohistochemistry (IHC) of the HER2 protein is the most common screening test, thus precise and reproducible $\mathrm{HC}$-scoring is of utmost importance. Interobserver variance still is a problem; in particular in gastric carcinomas the reliable differentiation of $\mathrm{HC}$ scores $2+$ and $1+$ is challenging. Herein we describe the physical basis of what we called the 'magnification rule': Different microscope objectives are employed to reproducibly subdivide the continuous spectrum of $\mathrm{HC}$ staining intensities into distinct categories $(1+, 2+, 3+)$.

Methods: HER2-IHC was performed on 120 breast cancer biopsy specimens ( $n=40$ per category). Width and color-intensity of membranous DAB chromogen precipitates were measured by whole-slide scanning and digital morphometry. Image-analysis data were related to semi-quantitative manual scoring according to the magnification rule and to the optical properties of the employed microscope objectives.

Results: The semi-quantitative manual HER2-IHC scores are correlated to color-intensity measured by image-analysis and to the width of DAB-precipitates. The mean widths \pm standard deviations of precipitates were: IHC-score $1+, 0.64$ $\pm 0.1 \mu \mathrm{m}$; score $2+, 1.0 \pm 0.23 \mu \mathrm{m}$; score $3+, 2.14 \pm 0.4 \mu \mathrm{m}$. The width of precipitates per category matched the optical resolution of the employed microscope objective lenses: Approximately $0.4 \mu \mathrm{m}(40 \mathrm{x}), 1.0 \mu \mathrm{m}(10 \mathrm{x})$ and $2.0 \mu \mathrm{m}(5 \times)$.

Conclusions: Perceived intensity, width of the DAB chromogen precipitate, and absolute color-intensity determined by image-analysis are linked. These interrelations form the physical basis of the 'magnification rule': $2+$ precipitates are too narrow to be observed with $5 \times$ microscope objectives, $1+$ precipitates are too narrow for $10 \times$ objectives. Thus, the rule uses the optical resolution windows of standard diagnostic microscope objectives to derive the width of the DAB-precipitates. The width is in turn correlated with color-intensity. Hereby, the more or less subjective estimation of IHC scores based only on the staining-intensity is replaced by a quasi-morphometric measurement. The principle seems universally applicable to immunohistochemical stainings of membrane-bound biomarkers that require an intensity-dependent scoring.
\end{abstract}

Keywords: HER2/neu, Immunohistochemistry, Breast cancer, Gastric cancer, Magnification rule, Predictive biomarker

\footnotetext{
*Correspondence: andreas.scheel@uk-koeln.de

${ }^{1}$ Institute of Pathology, University Hospital Cologne, Kerpener Str. 62, 50937

Cologne, Germany

Full list of author information is available at the end of the article
} 


\section{Background}

Targeting the HER2/neu pathway [1] has shown remarkable efficiency in the treatment of breast and gastric cancer [2,3]. A prerequisite for specific treatment is the demonstration of HER2 receptor overexpression by immunohistochemistry (IHC) and/or HER2/neu gene amplification by in-situ hybridization (ISH) [4-6]. Although advanced DNA-sequencing techniques have been demonstrated to analyze panels of oncogenic genomic aberrations including amplification of HER $2 /$ neu [7], current testing guidelines are based on IHC and ISH only [4, 5]. Most algorithms use IHC as first screening test and ISH as second test for the confirmation of equivocal cases (IHC 2+). Thus, IHC plays a key-role for HER2 testing in the routine diagnostics of breast and gastroesophageal cancer.

Interpretation of HER2-IHC is, however, more or less subjective which causes overall disagreement rates of around $10 \%$ [8]. The main issue in breast cancer is false positive scoring while in gastric cancer false negative scoring is the major problem. In a retrospective central review of 187 HER2 stained breast cancer specimens from 10 pathological institutions $9.5 \%$ of the negative cases were reclassified as positive and $31.7 \%$ of the positive cases as negative [9]. In gastric cancer, a central review of 394 HER2 stained specimens from 19 French pathological institutions revealed a false positive rate of $5 \%$ but a false negative rate of $27.4 \%$ [10]. This problem has recently also been addressed by the panelists of the new HER2 testing guideline for gastric and gastroesophageal cancer [5]. It is stated that in particular reproducibility of $1+$ and $2+$ IHC scores can be low and the distinction between $1+$ and $2+$ is "challenging". However, it remains unclear to the reader how this particular scoring problem can be resolved in clinical practice.

From the perspective of our long-standing experience with HER2 testing, e.g., as the central lab for HERA [2] and ToGA [3] trials, we consider subjectivity in IHC-scoring as major source of discordant results between local and central testing. This is particularly true for false negative HER2 testing in gastric cancer. In contrast to breast cancer where ring-shaped membranous staining is crucial to score a case either positive (IHC $3+$ ) or potentially positive (IHC2+), scoring in gastric cancer is solely based on intensity assessment by eye. Due to neurophysiological limitations it is practically impossible to objectively assess color-intensities alone unless other structural criteria, e.g. such as ring-shaped staining, are included [11-13].

In the context of the ToGA-study [3] we therefore developed a semiquantitative approach called 'magnification rule' (MR) that relates staining-intensity to the microscope magnification used to perceive it: Any membranous staining that can be recognized at low magnification (2.5-5x objective lens) corresponds to IHC3+; if higher magnification $(10 \times-20 \times)$ is needed to unequivocally identify stained membranes, $\mathrm{IHC} 2+$ is diagnosed. Any staining visible only at $40 \times$ objective lens represents an $\mathrm{IHC} 1+$ score $[14,15]$.

By using this rule the inter-observer consensus raised significantly from $\kappa<0.5$ to $\mathrm{\kappa}=0.805$ in a study on 547 gastric cancer specimens evaluated by six pathologists [15]. The finding was confirmed by a recent study which compared HER2 scoring by conventional light microscopy and by virtual microscopy and yielded interobserver concordance values of up to $\mathrm{K}=0.811$ [16]. Thus, the MR has already been incorporated in national recommendations on HER2-testing in gastric cancer [6, 17]. This quasi-morphometric semiquantitative approach applies also to HER2-IHC scoring in breast cancer where it is used for the first step of scoring, i.e. the estimation of the color-intensity, before the second criterion, the ring-shape pattern of the staining, is assessed $[15,17]$.

The present study analyses the physical background of the MR using a series of 120 breast cancer samples immunostained for HER2. The data provide a physical basis of how the MR works to overcome subjectivity in the scoring of membrane-bound IHC-biomarkers.

\section{Methods}

\section{Breast cancer biopsy specimens}

One hundred and twenty specimens of invasive breast carcinoma (no special subtype; NST) were retrospectively investigated using routinely HER2 stained biopsies diagnosed within one year at the Institute of Pathology Nordhessen, Kassel, Germany (Example photomicrographs: Fig. 1, Additional file 1: Figure S1). HER2 status was determined according to the 2013 updated ASCO/ CAP recommendations [4]. Accordingly, carcinomas classified as IHC 2+ were subsequently tested with dualcolor chromogenic in situ hybridization (ISH) for amplification of the HER2/Neu Gene (INFORM HER2 Dual ISH DNA Probe Cocktail Assay, Ventana Medical Systems Inc., Tucson, USA). Anonymized cases were scored by three pathologists and the consensus score for each taken as the final IHC HER2 status.

\section{IHC-staining and digital quantification}

Immunohistochemistry (IHC) was performed using the 4B5 anti-HER2 primary antibody and a polymer-based detection system (UltraView DAB) on a BenchMark automated staining system (all by Ventana Medical Systems Inc., Tucson, USA). Peroxidase-conjugated secondary antibodies were used for chromogenic detecting by oxidizing 3,3'-Diaminobenzidin according to the manufactures protocol. 


\begin{tabular}{|c|c|c|c|c|}
\hline Magnification & $40 x$ & $20 x$ & $10 x$ & $5 x$ \\
\hline $\begin{array}{l}\text { Consensus } \\
\text { Intensity-Score }\end{array}$ & $1+1$ & \multicolumn{2}{|c|}{$' 2+1$} & $13+1$ \\
\hline Num. Aperture & $0.65-0.75$ & $0.40-0.50$ & $0.25-0.30$ & $0.12-0.15$ \\
\hline Resolution $[\mu \mathrm{m}]$ & $0.40-0.46$ & $0.60-0.75$ & $1.0-1.20$ & $2.0-2.50$ \\
\hline $\begin{array}{l}\text { DAB-Precipitate } \\
\text { width } \pm S D[\mu \mathrm{m}]\end{array}$ & $0.64 \pm 0.1$ & \multicolumn{2}{|c|}{$1.0 \pm 0.23$} & $2.14 \pm 0.4$ \\
\hline
\end{tabular}
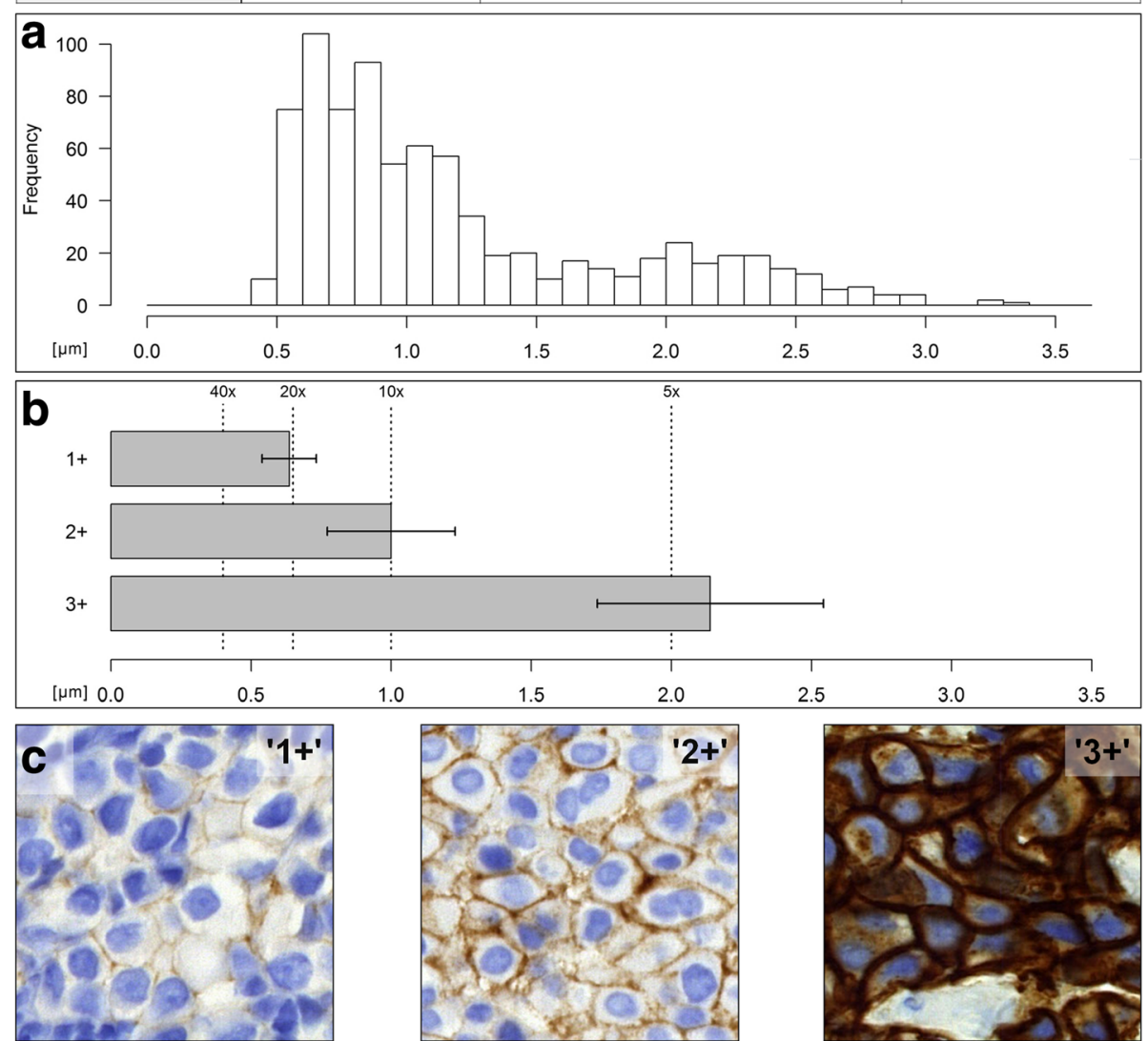

Fig. 1 Her2-IHC scoring categories reflect DAB-precipitate widths. Table: Microscope objectives have a fixed resolution that depends on the numerical aperture (Range: Values of common objectives). DAB-precipitates in HER2-HHC differ in width according to the intensity score. a histogram: Summary of 1200 DAB-precipitate-width measurements in $\mu \mathrm{m}$. b bar chart: Mean DAB-width (bars) \pm SD (antennae); resolution of standard microscope objectives (dashed lines). c images: Representative HER2-IHC stainings of invasive ductal breast carcinomas according to intensity score

IHC HER2 stained slides were digitized using a Pannoramic P250 whole slide scanner (3D Histech, Budapest, Hungary) at $5.11 \mathrm{pixel} / \mu \mathrm{m}$. DAB-precipitate thickness was measured with 'ImageJ' image-analysis software [18]. The regions of interest (ROIs) were manually defined according to the following rules: 10 non-adjacent tumor cells were measured per specimen. For each cell, ROIs perpendicular to the precipitate were drawn at 4 positions, i.e. 40 measurements per specimen, 4800 measurements in total. DAB-precipitate intensities were calculated using a color deconvolution algorithm [19]. The mean ROI-length and color intensity was calculated for each cell. The 8bit grey-scale intensity- values $(0=$ black, $256=$ white $)$ are as stated inverted, relative values to facilitate interpretation $(0 \%=$ white, no staining; $100 \%$ black, full staining-intensity).

\section{Microscope resolution}

The resolution (d) of the objective of a light microscope is the minimum distance required to distinguish two adjacent points on a focal plane. In light microscopy $d$ is determined by the numerical apertures (NA) of the microscope objectives and condenser and the wavelength of the employed light $(\lambda)$ through Abbe's law $=\frac{\lambda}{2 N A} \cdot N A$ is defined as $N A=n * \sin (\alpha)$ by the half-angle 
of the maximum cone of light that can pass through the objective $(\alpha)$ and the index of refraction $(n)$ of the medium in which the objective is used, $d=\frac{\lambda}{2 n \sin (\alpha)}$. For $\lambda=600 \mathrm{~nm}$, standard diagnostic microscope objectives yield the resolutions $5 \times: 2.0 \mu \mathrm{m}(N A=0.14), 10 \times: 1.0 \mu \mathrm{m}$ (NA=0.3), 20×: $0.6 \mu \mathrm{m}(N A=0.5), 100 \times: 0.4 \mu \mathrm{m}(N A=0.75)$.

\section{Statistics}

Statistics and statistical testing were performed using ' $R$ ' statistical programming language (http://www.r-project.org/ ). The data were found normally distributed and were tested using the Welch two-sample t-test. In all tests, the significance level was set to $\alpha=1 \%$.

\section{Results \\ HER2-IHC scoring categories reflect the width of DAB- precipitates}

In total, $n=120$ cases of invasive breast carcinoma (no special subtype; NST) were analyzed which yielded 4800 individual measurements. The linear DAB-precipitates formed by the HER2-IHC were quantified. Plotting the width of the precipitates per cells as continuous histogram yielded a biphasic distribution (Fig. 1a). However, if the cells per case are aggregated by the arithmetic mean, three groups emerged that matched the manual scoring categories (Fig. 1b, Additional file 2: Figure S2). The mean widths were found to be: IHC-score $1+, 0.64$ $\pm 0.1 \mu \mathrm{m}$; score $2+, 1.0 \pm 0.23 \mu \mathrm{m}$; score $3+, 2.14 \pm$ $0.4 \mu \mathrm{m}$. The differences between the three groups are statistically significant $(p<0.01)$. Thus, the scoring categories indicate groups of cases with perceivable differences in the widths of the DAB-precipitates.

The values were related to the optical resolutions of diagnostic microscope objectives. As predicted by the MR, precipitates of the scoring category $1+$ are too narrow to be observable with a $10 \times$ microscopic lens and are delineated best by a $40 \times$ objective. Moreover, $2+$ precipitates are broad enough to be visible at $10 \times$ but to narrow to be visible at $5 \times$. Only $3+$ precipitates were found broad enough to be readily recognizable if a $5 \times$ (or even a $2.5 \times$ ) objective lens is used (Fig. 1). The forth scoring category, ' 0 ' was omitted, as the DAB-precipitates were found absent or insufficient for quantification.

\section{Precipitate width and color intensity are correlated}

Color intensity of the DAB-precipitates was determined using color deconvolution [19]. Similar to the precipitate width, the intensities of the three scoring categories were significantly different $(p<0.01)$ (Fig. 2). Moreover, a good linear correlation between width and intensity was noticed among scoring categories $1+$ and 2+ (Pearson's $r=0.73$ ). The intensity in scoring category $3+$ was saturated (Additional file 3: Figure S3).
Precipitate width and color intensity do not differentiate between amplified and non-amplified cases in the IHC $2+$ scoring category

All cases classified as IHC $2+$ were subsequently tested for HER2/neu gene amplification by in situ hybridization (ISH). Among the study cases, 20 were ISH positive and 20 were ISH negative. The DAB-precipitate width showed a non-significant difference between the ISH positive cases $(1.02 \pm 0.23 \mu \mathrm{m})$ and the ISH negative cases $(0.98 \pm 0.22 \mu \mathrm{m}, p=0.02485)$. Indeed, histograms of the individual cells showed that the IHC $2+$ ISH positive and IHC 2+ ISH negative cases feature almost completely overlapping precipitate widths (Additional file 2: Figure S2). No difference in the HER2-IHC color intensities was noticed between ISH negative and ISH positive cases either ( $p=0.7493$ ) (Fig. 2).

\section{Discussion}

HER2-IHC scores determined according to the 'magnification rule' (MR) were compared to image-analysis of width and color intensity of the DAB chromogen precipitates along the tumor cell membranes. The parameters were closely correlated and matched the optical resolutions of the employed microscope objectives. This provides a physical basis of the MR which was originally established as an empirical rule for standardized HER2IHC scoring in gastric cancer.

HER2-IHC assays are based on peroxidase-coupled secondary antibodies that oxidize 3,3'-diaminobenzidine (DAB) into an insoluble, brownish precipitate at the spot of the bound epitope. As HER2 is confined to the cellmembrane, the reaction yields linear precipitates at the cell-boundaries. Technical aspects of HER2-IHC are robust and can be standardized by validated protocols, on-slide control tissue and external quality assessment [20-22] but interpretation of the resulting staining patterns may be challenging [4]: HER2-IHC scoring relies on subdividing the cases into categories based on stainingintensity $(0,1+$, negative; $2+$, equivocal, requires $\mathrm{ISH}-$ testing; 3+ positive) (Fig. 1, Additional file 1: Figure S1).

The human optical system is optimized to notice relative differences in color-intensity rather than absolute values. Visual stimuli are precortically processed in the retina through lateral inhibition which underlies varies optical illusions first described by Ernst Mach in 1865 as 'Mach bands' [12]. A given surface might appear brighter or darker depending on the luminosity of its surroundings $[11,13]$. Accordingly, intensity-scores in histopathology are in general prone to subjectivity.

This is of particular importance in HER2-IHC scoring in gastric cancer, which is based solely on stainingintensity. In contrast, HER2-IHC scoring in breast cancer also includes the staining-pattern as the DAB-precipitates have to be ring-shaped to be considered as IHC $3+$. In 

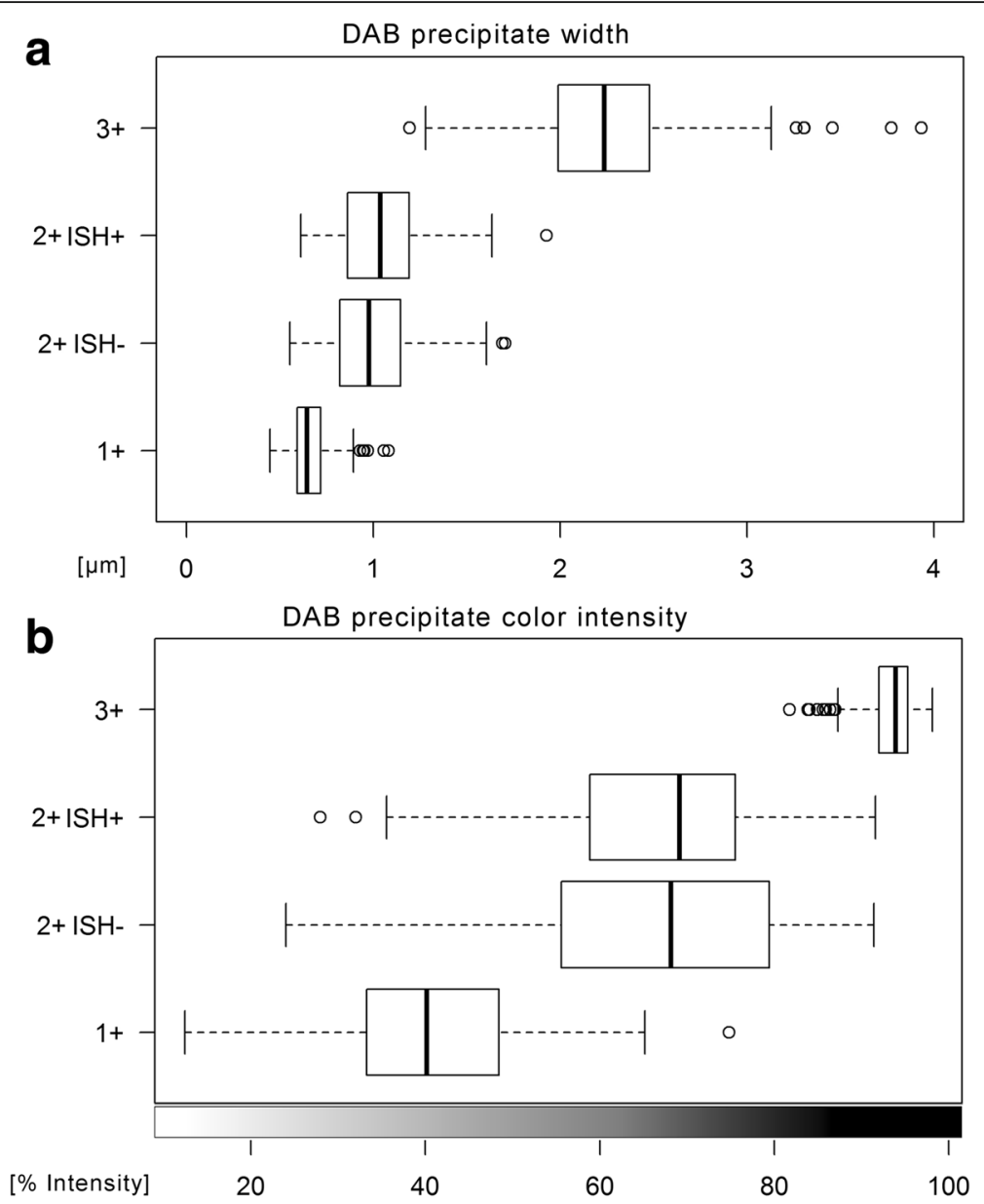

Fig. 2 Width and color intensity of HER2 are correlated. Immunohistochemistry of $n=120$ cases of invasive ductal carcinoma of the mammary gland show that width of the linear DAB precipitates (a) and DAB color intensity (b) are correlated (8bit scale, inverted relative values). However, neither parameter discriminates IHC Score 2+ samples that are Her2 amplified (ISH+) or not amplified (ISH-) by in situ hybridization

particular, inter-observer reproducibility of categories $1+$ and $2+$ scores can be low in gastric cancer. In two global clinical trials $6.4 \%(29 / 455)$ of cases with intestinal or mixed histology were found erroneously classified as HER2-IHC negative. In most of these cases $(n=21)$ initial scores were IHC 0 or IHC $1+$ whereas central countertesting revealed either IHC $2+$ and ISH positivity $(n=17)$ or even IHC $3+(n=4)$. Four additional cases locally IHC 2+ FISH-negative were centrally ISH positive [23].

In a recent French study comprising 393 centrally reevaluated gastric carcinomas false negative rate reached even $27.4 \%$ (20/73 HER2-IHC 2+). False positive rate was $5 \%(16 / 320)$ with an overall discordance rate of $9 \%$ [10].

In the present study it could be demonstrated that objectively measured widths and color intensities of the linear membranous precipitates correlate with the semiquantitative intensity score manually assessed by MR. Utilizing this approach circumvents the need to interpret the staining just by color-intensity and constitutes a quasi-morphometric measurement.
Our data suggest that the MR might be applicable to other membrane-bound biomarkers as well. Indeed, interobserver concordance of IHC-scoring of EGFR could be significantly improved within a round-robin test that included 11 international pathological laboratories [24]. The MR could also be included in the comprehensive 'Histo-Score' (H-Score) [25]. The H-Score incorporates all IHC-intensity categories and is frequently used to determine an optimal cut-point in IHC-scoring [26, 27]. The prerequisite to using the MR is that biological relevant scoring-categories have to be reflected by differences in the geometry of the histological stain that match the optical resolution windows of the microscope objectives. A given IHC-protocol could be optimized to match the appropriate intensity range.

The interrelation of DAB-width, -intensity and score might also form the basis for an image-analysis algorithm which mimics the magnification rule. Different approaches have been investigated for HER2-IHC imageanalysis by using color intensities $[28,29]$ or geometric 
properties of the staining pattern [30]. Recent advances in digital image analysis have shown to increase of interobserver agreement and decrease of the number of equivocally scored cases $[31,32]$.

\section{Conclusions}

IHC scoring by using the 'magnification rule' is a semiquantitative procedure that circumvents neurophysiological restrictions of our visual system. It is based on physical interrelations and can be used to overcome subjectivity in HER2 IHC-testing, particularly in gastric cancer. It might also be applicable to other membranebound IHC-stainings. As a practical and easy-to-use method it has found wide application and was incorporated into national and international recommendation on HER2-IHC $[6,15,17]$.

\section{Additional files}

Additional file 1: Figure S1. Example photomicrographs of HER2-IHC. Images depict scoring categories $1+, 2+$ and $3+$ at magnifications reflecting different microscope objectives (2.5x - 63x. Inserts: Magnified details, $4 \times$ additional magnification). Note that the linear DAB-precipitates in categories $1+$ and $2+$ are not perceivable at low power magnification $(2.5 \times, 5 \times)$. (TIFF $47314 \mathrm{~kb}$ )

Additional file 2: Figure S2. Width of HER2 DAB-precipitates and result of in situ hybridization (ISH). Histograms of $n=1200$ measurements in 40 cases per scoring category; estimated density (graphs). (TIFF 1103 kb)

Additional file 3: Figure S3. Scatter-plot of HER2 DAB-precipitates width and color intensity. For scoring intensities $1+$ and $2+$ (grey), width and intensity show a linear correlation ( $r=0.73$, dashed lined). Scoring category $3+$ shows saturated intensity $(n=1200$ measurements in 40 cases per scoring category). (JPEG $585 \mathrm{~kb}$ )

\section{Abbreviations \\ ASCO/CAP: American Society of Clinical Oncology / College of American Pathologists; DAB: Diaminobenzidine; HER2: The HER2 protein, i.e. human epidermal growth factor receptor 2; HER2/neu: The HER2/neu gene which encodes the HER2 protein; HERA: Acronym of the clinical phase 3 trial that tested adjuvant Trastuzumab in breast cancer, cf. Lancet. 2017; 389:1195- 1205; IHC: Immunohistochemistry; ISH: In-situ hybridization; \\ MR: Magnification rule; NA: Numerical aperture; ROI: Region of interest; SD: Standard deviation; ToGA: Acronym of the clinical phase 3 trial that tested adjuvant Trastuzumab in gastric cancer, cf. Lancet. 2010; 376:687-97; 0, $1+, 2+, 3+$ : Categories of the scoring system for HER2-IHC interpretation; 4B5: Clone of the primary anti-HER2 antibodies used for HER2-IHC}

\section{Acknowledgements}

We are much obliged to Sysmex (Norderstedt, Germany) for kindly providing us with the Pannoramic P250 whole slide scanner. We would like to thank Ulrike Hampacher and Heike Fliegel (Institute of Pathology Nordhessen, Kassel) for excellent technical assistance.

\section{Funding}

The study did not receive funding.

\section{Availability of data and materials}

Please contact author for data requests.

\section{Authors' contributions}

The magnification rule (MR) was conceived by JR. Usage of the MR in practical HER2-testing was tested and discussed by FPL, WH, GB, PM, MH, BJ and JR. The study was designed by AHS and JB under guidance of JR, PM and $\mathrm{MH}$ with support of FPL, WH and GB. Whole slide scanning was performed by JB and
AHS. Manual HER2-scoring was done by JR, MH and PM. Image-analysis was done by AHS. Data-analysis was done by AHS under guidance of JR, PM, and BJ. The manuscript was drafted by AHS and JR. The manuscript was discussed with all coauthors and revised accordingly by AHS. The final version of the manuscript was agreed by all authors.

\section{Ethics approval and consent to participate}

The study was approved by the responsible local ethical committee of the Landesärztekammer Hess, Frankfurt, Germany (file number FF 135/2013). The committee decided that additional informed consent was not required for this retrospective analysis.

\section{Consent for publication}

Not applicable.

\section{Competing interests}

The authors declare that they have no competing interests.

\section{Publisher's Note}

Springer Nature remains neutral with regard to jurisdictional claims in published maps and institutional affiliations.

\section{Author details}

${ }^{1}$ Institute of Pathology, University Hospital Cologne, Kerpener Str. 62, 50937 Cologne, Germany. ${ }^{2}$ Département de Pathologie, Centre Jean-Perrin, 58, rue Montalembert, 392, 63011 Clermont-Ferrand cedex 1, BP, France.

${ }^{3}$ Department of Laboratory Medicine and Pathobiology, University of Toronto, Toronto, Canada. ${ }^{4}$ Institute of Pathology, University Hospital Dresden, Fetscherstr, 74, 01307 Dresden, Germany. ${ }^{5}$ Institute of Pathology Nordhessen, Germaniastraße 7, 34119 Kassel, Germany. ${ }^{6}$ Institute of Pathology, University Hospital Göttingen, Robert-Koch-Str. 40, 37075 Göttingen, Germany. ${ }^{7}$ Targos Molecular Pathology GmbH, Germaniastraße 7, 34119 Kassel, Germany.

Received: 15 October 2017 Accepted: 1 March 2018 Published online: 12 March 2018

References

1. Yarden $Y$, Pines $G$. The ERBB network: at last, cancer therapy meets systems biology. Nat Rev Cancer. 2012;12:553-63.

2. Cameron D, Piccart-Gebhart MJ, Gelber RD, et al. 11 years' follow-up of trastuzumab after adjuvant chemotherapy in HER2-positive early breast cancer: final analysis of the HERceptin adjuvant (HERA) trial. Lancet. 2017; 389:1195-205.

3. Bang YJ, Van Cutsem E, Feyereislova A, et al. Trastuzumab in combination with chemotherapy versus chemotherapy alone for treatment of HER2positive advanced gastric or gastro-oesophageal junction cancer (ToGA): a phase 3, open-label, randomised controlled trial. Lancet. 2010;376:687-97.

4. Wolff AC, Hammond ME, Hicks DG, et al. Recommendations for human epidermal growth factor receptor 2 testing in breast cancer: American Society of Clinical Oncology/College of American Pathologists clinical practice guideline update. J Clin Oncol. 2013;31:3997-4013.

5. Bartley AN, Washington MK, Ismaila N, Ajani JA. HER2 testing and clinical decision making in gastroesophageal adenocarcinoma: guideline summary from the College of American Pathologists, American Society for Clinical Pathology, and American Society of Clinical Oncology. J Oncol Pract. 2017;13:53-7.

6. Baretton G, Dietel M, Gaiser T, et al. HER2 testing in gastric cancer : results of a meeting of German experts. Pathologe. 2016;37:361-6.

7. Ross DS, Zehir A, Cheng DT, et al. Next-generation assessment of human epidermal growth factor receptor 2 (ERBB2) amplification status: clinical validation in the context of a hybrid capture-based, comprehensive solid tumor genomic profiling assay. J Mol Diagn. 2017;19:244-54.

8. Piccart-Gebhart MJ. St.Gallen International Breast Cancer Conference Primary Therapy of Early Breast Cancer Evidence, Controversies, Consensus; 11 - 14 Mar 2009; St. Gallen, Switzerland.

9. Orlando L, Viale G, Bria E, et al. Discordance in pathology report after central pathology review: implications for breast cancer adjuvant treatment. Breast. 2016;30:151-5.

10. Monges $G$, Terris $B$, Chenard M-P, et al. Assessment of HER2 status from an epidemiology study in tumor tissue samples of gastric and gastro- 
esophageal junction cancer: Results from the french cohort of the HEREAGLE study. JCO. 2013;31(4, Supplement S):26. http://ascopubs.org/doi/ abs/10.1200/jco.2013.31.4_suppl.26.

11. Goldstein EB, editor. Blackwell handbook of perception. 4th ed. USA: Blackwell Publishers Inc; 2001. p. 53 ff.

12. Lotto RB, Williams SM, Purves D. Mach bands as empirically derived associations. Proc Natl Acad Sci U S A. 1999;96:5245-50.

13. Adelson EH. Perceptual organization and the judgment of brightness. Science. 1993;262:2042-4.

14. Rüschoff J, Dietel M, Baretton $G$, et al. HER2 diagnostics in gastric cancerguideline validation and development of standardized immunohistochemical testing. Virchows Arch. 2010;457:299-307.

15. Rüschoff J, Hanna W, Bilous M, et al. HER2 testing in gastric cancer: a practical approach. Mod Pathol. 2012;25:637-50.

16. Behrens HM, Warneke VS, Böger C, et al. Reproducibility of Her2/neu scoring in gastric cancer and assessment of the 10\% cut-off rule. Cancer Med. 2015:4:235-44.

17. Rakha EA, Starczynski J, Lee AH, Ellis IO. The updated ASCO/CAP guideline recommendations for HER2 testing in the management of invasive breast cancer: a critical review of their implications for routine practice. Histopathology. 2014;64:609-15.

18. Schneider CA, Rasband WS, Eliceiri KW. NIH image to ImageJ: 25 years of image analysis. Nat Methods. 2012;9:671-5.

19. Ruifrok AC, Johnston DA. Quantification of histochemical staining by colour deconvolution. Anal Quant Cytol Histol. 2001;23:291-9.

20. Choritz H, Büsche $G$, Kreipe $H$, et al. Quality assessment of HER2 testing by monitoring of positivity rates. Virchows Arch. 2011;459:283-9.

21. Vyberg M, Nielsen S, Røge R, et al. Immunohistochemical expression of HER2 in breast cancer: socioeconomic impact of inaccurate tests. BMC Health Serv Res. 2015;15:352.

22. Rüschoff J, Lebeau A, Kreipe $\mathrm{H}$, et al. Assessing HER2 testing quality in breast cancer: variables that influence HER2 positivity rate from a large, multicenter, observational study in Germany. Mod Pathol. 2017:30:217-26.

23. Cunningham D, Shah MA, Smith D, et al. False-negative rate for HER2 testing in 738 gastric and gastroesophageal junction cancers from two global randomized clinical trials. J Clin Oncol. 2015;33(Supplement 3):16. http://ascopubs.org/doi/abs/10.1200/jco.2015.33.3_suppl.16.

24. Rüschoff J, Kerr KM, Grote HJ, et al. Reproducibility of immunohistochemical scoring for epidermal growth factor receptor expression in non-small cell lung cancer: round robin test. Arch Pathol Lab Med. 2013;137:1255-61.

25. Hirsch FR, Varella-Garcia M, Bunn PA Jr, et al. Epidermal growth factor receptor in non-small-cell lung carcinomas: correlation between gene copy number and protein expression and impact on prognosis. J Clin Oncol. 2003:21:3798-807.

26. Garon EB, Rizvi NA, Hui R, et al. Pembrolizumab for the treatment of nonsmall-cell lung cancer. N Engl J Med. 2015;372:2018-28.

27. Dolled-Filhart M, Roach C, Toland G, et al. Development of a companion diagnostic for Pembrolizumab in non-small cell lung cancer using immunohistochemistry for programmed death Ligand-1. Arch Pathol Lab Med. 2016:140:1243-9.

28. Ali HR, Irwin $M$, Morris $L$, et al. Astronomical algorithms for automated analysis of tissue protein expression in breast cancer. Br J Cancer. 2013;108:602-12.

29. Jeung J, Patel R, Vila L, Wakefield D, Liu C. Quantitation of HER2/neu expression in primary gastroesophageal adenocarcinomas using conventional light microscopy and quantitative image analysis. Arch Pathol Lab Med. 2012;136:610-7.

30. Laurinaviciene A, Dasevicius D, Ostapenko V, Jarmalaite S, Lazutka J, Laurinavicius A. Membrane connectivity estimated by digital image analysis of HER2 immunohistochemistry is concordant with visual scoring and fluorescence in situ hybridization results: algorithm evaluation on breast cancer tissue microarrays. Diagn Pathol. 2011;6:1746-596.

31. Helin HO, Tuominen VJ, Ylinen O, Helin HJ, Isola J. Free digital image analysis software helps to resolve equivocal scores in HER2 immunohistochemistry. Virchows Arch. 2016;468:191-8.

32. Nielsen SL, Nielsen S, Vyberg M. Digital image analysis of HER2 Immunostained gastric and gastroesophageal junction adenocarcinomas. Appl Immunohistochem Mol Morphol. 2017;25:320-8.

\section{Submit your next manuscript to BioMed Central and we will help you at every step:}

- We accept pre-submission inquiries

- Our selector tool helps you to find the most relevant journal

- We provide round the clock customer support

- Convenient online submission

- Thorough peer review

- Inclusion in PubMed and all major indexing services

- Maximum visibility for your research

Submit your manuscript at www.biomedcentral.com/submit
Biomed Central 